\title{
EDITORIAL
}

\section{Three decades of antiviral drugs}

\author{
Thirty years ago, just three drugs were available for the treatment of viral infections, \\ but now more than forty have been approved. What have been the guiding principles in \\ these achievements and what might the future hold for antiviral drug development?
}

In the late 1970s, the discovery that acyclic nucleoside analogues, in particular acyclovir, could inhibit DNA replication of herpes simplex virus (HSV) at concentrations far below those that affect cellular DNA synthesis sparked a new era in antiviral chemotherapy. The underlying reason for the selectivity - that acyclovir is specifically converted to the active metabolite by an HSV-encoded thymidine kinase - was unexpected, but the potential for exploiting viral enzymes to develop potent and specific antiviral drugs was clearly demonstrated. Acyclovir subsequently became a successful treatment for HSV-1 and HSV-2.

The success of acyclovir provided encouragement in the early 1980s in the face of a new viral pathogen: human immunodeficiency virus (HIV, then called LAV-1/ HTLV-III), which was identified as the (then tentative) cause of AIDS, leading to an intensive search for inhibitors of this retrovirus. The most successful of the anti-HIV agents discovered in this early period, and also the first to be licensed for clinical use, was azidothymidine (AZT), which emerged from a screening effort of around 100 nucleoside analogues selected by Wellcome Laboratories and evaluated by the National Cancer Institute. Again, the selectivity of the inhibitory activity of AZT for a viral enzyme - HIV reverse transcriptase - compared with enzymes involved in DNA synthesis in normal cells provided a therapeutic window for its use.

Two key advances in the 1980s - genome sequencing and structure-based drug design - then facilitated a new approach to targeting viral enzymes, guided by rational design. Efforts to elucidate the HIV life cycle, aided by the genome sequence of the virus, revealed that a virally encoded aspartyl protease was crucial in viral replication. Specific design of protease inhibitors based on the transition-state peptidomimetic principle - replacement of the peptide linkage by a non-cleavable bond - and aided by structures of the protease ultimately led to the introduction of HIV protease inhibitors in the mid-1990s. The combination of these drugs with nucleoside reverse transcriptase inhibitors began the era of highly active antiretroviral therapy (HAART), transforming HIV infection from a death sentence to a chronically manageable disease.

This success was not the end of the story in the battle against HIV, as resistance to anti-HIV drugs emerges, often rapidly. To combat this, new agents in existing classes and new classes of drugs from both more serendipitous screening and rational design have been developed. Twenty-four anti-HIV drugs have received regulatory approval: eleven are targeted at the HIV reverse transcriptase and ten are targeted at the HIV protease, the others target HIV cell entry, HIV cell fusion and HIV integrase (Supplementary information S1 (table); available online).

Meanwhile, the development of drugs against other viruses has also progressed considerably, mirroring some of the themes seen in the development of anti-HIV drugs. For example, in the early 1990s, 'transition-state' mimicry also inspired the design of influenza virus neuraminidase (also known as sialidase) inhibitors, which are currently the mainstay of pharmacological defences against an influenza pandemic. Also, the compounds and treatment strategies developed for HIV could be considered as a paradigm for the development of similar approaches for the treatment of other viral infections, in particular hepatitis $\mathrm{C}$ virus (HCV). Although $\mathrm{HCV}$ is fundamentally different to HIV, several classes of drugs targeting viral enzymes - in this case HCV RNA replicase and $\mathrm{HCV}$ serine protease - might one day be combined in a way similar to those targeting HIV reverse transcriptase and the HIV aspartyl protease. Importantly, for HCV, combination of antiviral drugs with immunomodulatory interferons may eradicate the infection, something that still seems a distant goal for HIV therapy.

Although HIV and HCV are currently the major focus of antiviral drug research, this should not detract from the importance of developing and implementing drug therapies for several other virus infections, including hepatitis B virus and influenza virus. Severe acute respiratory syndrome (SARS) virus and other respiratory virus infections that may (re)-emerge at any time, and haemorrhagic fever virus (such as Ebola) and encephalitis virus (such as West Nile) infections, also merit serious attention.

The ongoing need to combat existing and emerging viral infections calls for continued collaboration between medicinal chemists and biomedical researchers to identify and develop new therapies, harnessing both 'serendipitous' screening strategies and rational design, as exemplified in the discovery of acyclovir and the HIV protease inhibitors. And finally, from a global health perspective, continued efforts to make these therapies available to all those that need them are highly warranted.

Erik De Clercq 